\title{
A Modular Low-Complexity ECG Delineation Algorithm for Real-Time Embedded Systems
}

\author{
José Manuel Bote, Joaquín Recas ${ }^{(0)}$, Francisco Rincón, David Atienza, Fellow, IEEE, \\ and Román Hermida, Senior Member, IEEE
}

\begin{abstract}
This work presents a new modular and lowcomplexity algorithm for the delineation of the different ECG waves (QRS, P and T peaks, onsets, and end). Involving a reduced number of operations per second and having a small memory footprint, this algorithm is intended to perform realtime delineation on resource-constrained embedded systems. The modular design allows the algorithm to automatically adjust the delineation quality in runtime to a wide range of modes and sampling rates, from a ultralow-power mode when no arrhythmia is detected, in which the ECG is sampled at low frequency, to a complete high-accuracy delineation mode, in which the ECG is sampled at high frequency and all the ECG fiducial points are detected, in the case of arrhythmia. The delineation algorithm has been adjusted using the QT database, providing very high sensitivity and positive predictivity, and validated with the MIT database. The errors in the delineation of all the fiducial points are below the tolerances given by the Common Standards for Electrocardiography Committee in the high-accuracy mode, except for the $P$ wave onset, for which the algorithm is above the agreed tolerances by only a fraction of the sample duration. The computational load for the ultralow-power 8-MHz TI MSP430 series microcontroller ranges from $0.2 \%$ to $8.5 \%$ according to the mode used.
\end{abstract}

Index Terms-Electrocardiogram, delineation, real-time, energy-constrained systems, wearable medical devices.

\section{INTRODUCTION}

$\mathbf{T}$ HE ECG signal, which represents the electrical activity of the heart, is an essential tool to monitor the status of the heart and evaluate its condition. Most of the techniques for the

Manuscript received May 10, 2016; revised December 15, 2016 and February 2, 2017; accepted February 8, 2017. Date of publication February 17, 2017; date of current version March 5, 2018. This work was partially supported by the EU (FEDER) and the Spanish Ministry of Economy and Competitiveness under Grant TIN2015-65277-R and by the ObeSense (no. 20NA21 143081) RTD project, evaluated by the Swiss NSF and funded by Nano-Tera.ch with Swiss Confederation financing, as well as the NCCR Robotics-Sub-project 9.2: Bidirectional smart jacket.

J. M. Bote, J. Recas, and R. Hermida are with the Department of Computer Architecture and Automation, Complutense University of Madrid, 28040 Madrid, Spain (e-mail: jmbrosado@ucm.es; recas@ucm.es; rhermida@ucm.es).

F. Rincón is with SmartCardia, 1015 Lausanne, Switzerland (e-mail: francisco.rincon@smartcardia.com).

D. Atienza is with the School of Engineering, Ecole Polytechnique Federale de Lausanne, 1015 Lausanne Switzerland (e-mail: david.atienza @ epfl.ch).

Digital Object Identifier 10.1109/JBHI.2017.2671443 automatic analysis of the ECG signal rely on features derived from its characteristic points. Therefore, an accurate detection of the peaks and boundaries of the different ECG waves is vital to achieve a reliable automatic diagnosis of cardiac issues. In many situations, such as ambulatory monitoring, a real-time automated system which can extract or delineate the different ECG waves is useful.

The first techniques applied to delineate the QRS complex in the ECG signal were based in the ECG amplitude and its first derivative. Although some of these implementations, for instance [1], are quite efficient and feature high sensitivity and specificity, they are limited to the detection of the QRS complex. However, in order to perform an accurate ECG analysis, the peak and boundaries of the $\mathrm{P}$ and $\mathrm{T}$ waves are also required. Nowadays, a plethora of more advanced methods to delineate all these fiducial points have been developed. These methods include the use of wavelets (the most widely used technique, even for detecting myocardial scars studying the ECG waves morphologies [2]) [3]-[5], the Hilbert transform [6], the fasor transform [7], dynamic time warping [8], artificial neural networks [9], hidden Markov models [10], time-domain morphology and gradient based algorithms [11] or morphological transforms [12].

These new approaches involve a high computational cost due to their complexity. If the delineation is executed off-line or without any restriction of time, they can be good approximations to medical annotations. However, the increasing use of miniaturized embedded wearable devices for real-time health monitoring and diagnosis, is driving the need for more efficient algorithms that can be adapted to the low processing capabilities and energy budget of these devices, while achieving the high accuracy required by medical standards. Some of these devices have already reached the market, like Corventis NUVANT Mobile Cardiac Telemetry System [13], Vital Connect HealthPatch MD [14] or Preventice BodyGuardian Remote Monitoring System [15]. However, they are limited to ECG streaming or very simple processing, to extract key parameters such as heart beat rate.

Currently, the wearables market is steadily growing up, developing new devices that are able to monitor heart rate (HR) 24/7, using GPS for running or monitoring sleep patterns and quality. Fitness trackers or smartwatches can be used to record and/or analyze real time signals without anything but a sensor mounted on the device or a chest strap. In case of HR, devices such as the wrist-based Fitbit models [16] (Charge 2, Blaze or 
Surge) or the Garmin vívo series [17], the HR is measured optically and have an autonomy between 5 to 8 days. On the other hand, wearables like Polar M400 [18] measure HR electrically with a chest strap and a smartwatch.

New devices arrive at the market every year, as the Cronovo [19] ECG monitor watch, that detects stress level, heart rate variability, fatigue level, automatic SOS alerts or drowsiness alerts, or the QardioCore [20], a 3-lead wireless ECG monitor, clinically validated, that uses $200-\mathrm{Hz}$ sampling rate and 16-bit resolution, with an autonomy of 2 days. The proposed algorithm for real-time ECG delineation will help to enable the nextgeneration of wearable monitoring systems, that can rely on the delineation results to perform online on-board arrhythmia detection.

In an effort to achieve a suitable algorithm for these wearable embedded systems, [21] proposed a real-time wavelet-based delineator, also making emphasis in the importance of embedded signal filtering to compensate the different sources or noise and artifacts that appear during ambulatory monitoring. The work we present in this paper goes one step further, proposing a similar structure as the algorithm for heartbeat classification presented in [22], where a simple and energy efficient classification algorithm is running all the time, to identify which parts of an ECG are critical, and activate, only in those cases, detailed (and more computationally intensive) diagnosis algorithms; and as in [23] where filters and thresholds are used in the detection of the ECG fiducial points for predicting ventricular arrhythmias in their real-time hardware implementation. Therefore, this paper proposes a new delineation algorithm that is not only less computationally complex and more energy efficient than stateof-the-art techniques, but also much more flexible and modular, being able to always achieve an optimal trade-off between energy consumption and delineation accuracy, depending on the available energy budget and the required performance, to carry out a precise arrhythmia detection.

The simple yet accurate delineation algorithm presented in this paper is based on the second derivative of a low-pass FIR filtered ECG signal whose cut-off frequency is $14 \mathrm{~Hz}$. Noise reduction, using another low-pass FIR filter with frequency of $40 \mathrm{~Hz}$, and baseline wander removal, applying different morphological filters, are also considered. The advantages of the proposed algorithm in comparison to the previous work [21] are the following:

1) In the delineation algorithm presented in this work, the sampling rate is adjusted in run time to reduce energy consumption in terms of CPU processing and ADC usage. The wavelet techniques require either redesigning the filter banks or resampling the input signal, inducing errors [5], while the presented algorithm only needs to adjust the FIR filters coefficients to maintain their cut-off frequencies (the coefficients can be computed offline and stored in a Flash for a certain set of frequencies).

2) The presented technique is able to detect a beat with almost no delay, allowing the ADC to be turned off until the next beat is expected allowing the system to save energy. Conversely, the wavelet transform uses a sliding

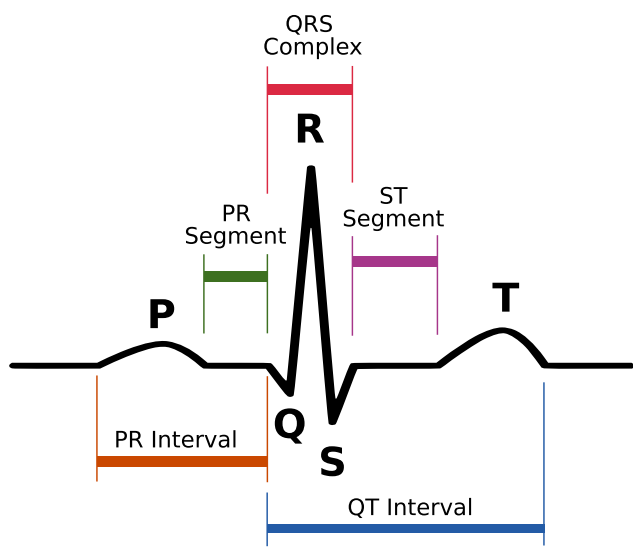

Fig. 1. Normal ECG. Schematic diagram of normal sinus rhythm for a human heart as seen on ECG. (Wikimedia Common, Public Domain.)

window to detect beats, and therefore all the samples within it are needed.

3) If a complete delineation is not needed, e.g. the heart rate is regular and the subject has no pathologies, the modularity of the presented algorithm allows some parts to be disabled, highly reducing the computational load. The system can also enable all the modules automatically if a given heart condition, e.g. a tachycardia or bradycardia, is detected. In the wavelet method, most of the CPU capacity is used to compute the scales and, as the R peak detection uses all of them, not detecting the rest of the fiducial points would not reduce significantly the CPU burden.

The rest of the paper is organized as follows. Section II presents the modular design of the delineation algorithm proposed in this work, including a case of study in which the algorithm automatically changes the delineation quality in response to heart rate variations. Then, in Section III, the implementation of the algorithm on MSPSim is described, and its performance evaluation is provided, as well as a comparative study with other state-of-the-art techniques. Finally, the conclusions of this work are summarized in Section IV.

\section{ECG Delineation Algorithm}

The ECG signal presents different waves, each of them related to a physiological phase of the cardiac cycle. As shown in Fig. 1, a heart beat can be split into three main parts: the $\mathrm{P}$ wave, the QRS complex and the T wave. The $\mathrm{P}$ wave indicates the start of a beat and represents the atrial depolarization. The QRS complex is a group of three consecutive waves and corresponds to the ventricular depolarization. Finally, the $\mathrm{T}$ wave indicates the ventricular repolarization.

In this section, a new modular algorithm to detect different ECG waves (P, QRS complex and T) and their fiducial points (peak, onset and end) is described. The detection algorithm of the ECG waves is summarized as follows (see Fig. 2):

1) The delineation algorithms begins with a QRS peak detection. 


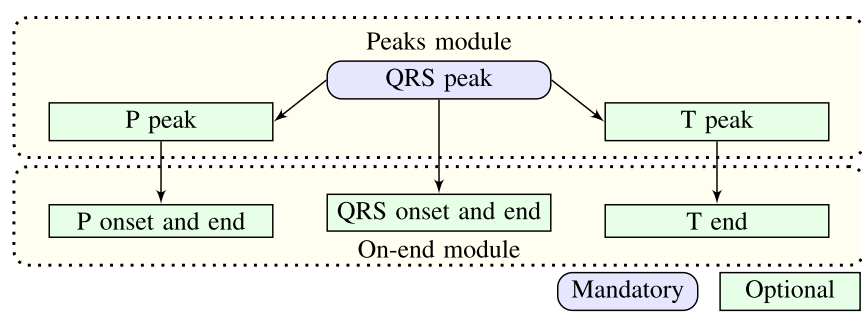

Fig. 2. Conceptual flowchart of ECG delineation.

2) Once a QRS peak has been detected, its boundaries (onset and end) can be found, if needed, before and after that QRS peak (this defines the QRS complex).

3) Also P and T peaks may be searched before and after that QRS complex, with their onset and end.

When the raw ECG signal is acquired, high-frequency noise, muscle motion artifacts and baseline wander can be present. These perturbations need to be removed before applying the delineation algorithm, to ensure good accuracy in the detection of the fiducial points. Fig. 3 shows the flowchart of ECG signal processing used in the delineation. The Peaks module (left) is used for detecting the QRS peak and, optionally, the P and T peaks. The On-end module (right) can be used for delineating the onsets and ends for the ECG waves if needed. The Onend module is subdivided into three completely-independent modules: the QRS-on-end module, the P-on-end module and the T-end module that can be enabled or disabled as required.

\section{A. Peaks Module}

The purpose of this module is to detect the QRS peaks and, also, the $\mathrm{P}$ and $\mathrm{T}$ peaks if needed. In order to do this, a lowpass FIR ${ }^{1}$ filter with a cut-off frequency of $14 \mathrm{~Hz}$ is applied to the $E C G_{\text {raw }}$ signal. With this aggressive filter, any type of high-frequency signal is eliminated, while $\mathrm{P}, \mathrm{QRS}$ and $\mathrm{T}$ wave peak positions are preserved, see $E C G_{L P 14}$ signal in Fig. 4. Thus $E C G_{L P 14}$ is used to find the peaks of the P, T waves and QRS complexes, but it is too aggressive to detect smaller less energetic waves, like $\mathrm{Q}$.

Although IIR filters can achieve filtered signals using less taps, memory and operations than FIR filters, they are more susceptible to numerical stability problems. Therefore, FIR filters have been chosen because they are easier to implement in real-time (lower complexity) and their stability is assured, although more samples are needed for processing the signal and a bigger delay is introduced (a N-order FIR filter produces a delay of N/2 samples).

The delineation of the QRS peak consists in detecting the most significant peak of the $E C G_{L P 14}$ signal. Since the shape of the QRS complex depends on the orientation of the selected lead, the most significant peak is an $\mathrm{R}$ wave in some leads (e.g. V6) or an S wave in other leads (e.g. V1), where a small R peak is followed by a large negative deflection ( $\mathrm{S}$ wave). In this paper,

\footnotetext{
${ }^{1}$ Different windows to implement the FIR filter have been studied (e.g. Hamming, Blackman, etc.) and the results provided are similar. In this paper, a Blackman window has been chosen although other windows may be used.
}

we define the former QRS complexes as positives and the latter as negatives.

To detect the peak of the QRS complex, the first and second derivatives are computed. As shown in Fig. 5(a) for positive QRS peaks, the second derivative is always negative and its value is larger (in absolute value) near the peak than in the rest of the beat, and the first derivative crosses the zero line. In case of negative QRS peaks, the second derivative is always positive. A normal ECG is depicted in Fig. 6, containing positive and negative $\mathrm{QRS}$ complexes.

The first step in the QRS peak detection is to select a filtered ECG signal excerpt (from $E C G_{L P 14}$ ) that is big enough to contain at least one beat; this excerpt is stored in a 2-second circular buffer (also called window) and updated every new ECG sample. The length of 2 seconds for the buffer allows $\mathrm{HR}$ as low as 30 beats per minute. Every time the 2-second window (or circular buffer) is completed, the maximum and the minimum of the second derivative are found $\left(\max \left(E C G_{L P 14}^{\prime \prime}\right)\right.$ and $\min \left(E C G_{L P 14}^{\prime \prime}\right)$ respectively) to compute a value that will be used later. Finally, a moving average $\left(A v g_{Q R S}\right)$ of those last 5 values will be used later to calculate a threshold to detect QRS peaks; this threshold is updated every time that a new window is completed.

A QRS peak is detected within the circular buffer when the following conditions (evaluated with the arrival of each new ECG sample) are met at a point:

1) The sign of the first derivative of $E C G_{L P 14}$ has to change (marked as $E C G_{L P 14, s c}^{\prime}$ in Fig. 3). This means that there is a local maximum or minimum in the filtered ECG signal.

2) The magnitude (absolute value) of the second derivative of $E C G_{L P 14}$ has to be greater than the threshold $Q R S_{\text {th }}=Q R S_{\text {factor }} \cdot A v g_{Q R S}$, where $Q R S_{\text {factor }}=$ 0.33 will be justified later, and $A v g_{Q R S}$ was previously defined as the moving average of the last five minimum/maximum window values and, therefore, $R_{t h}$ is updated every 2 seconds (every time $A v g_{Q R S}$ is updated due to a new 2-second window is completed) to adapt the algorithm to a new ECG excerpt dynamically. It should be noted that $Q R S_{\text {factor }}$ has meaning between 0 and 1 .

3) The time difference between two consecutive QRS peaks has to be greater than $250 \mathrm{~ms}$. This time interval allows to detect heart rates as high as 240 beats per minute.

The conditions for detecting a QRS peak are evaluated for the arrival of each new ECG sample. It should be noted that the FIR filters, morphological filters and derivatives introduce a known delay between raw and processed samples that is taken into account. Once a QRS peak is detected, the $\mathrm{P}$ and $\mathrm{T}$ peaks are delineated if needed, searching them between 2 consecutive QRS peaks (the $\mathrm{T}$ wave belongs to the previous beat and the $\mathrm{P}$ wave, to the current).

The $\mathrm{P}$ wave is the first wave in a beat. It is shorter than $120 \mathrm{~ms}$ in normal patients and it may be increased, for instance, in patients with history of atrial fibrillations, up to 170 ms [24]. Also, the PR interval (see Fig. 1) is between $120 \mathrm{~ms}$ and $200 \mathrm{~ms}$ [25]. For this reasons, the delineation algorithm looks for a possible P peak in a time window between $100 \mathrm{~ms}$ and 


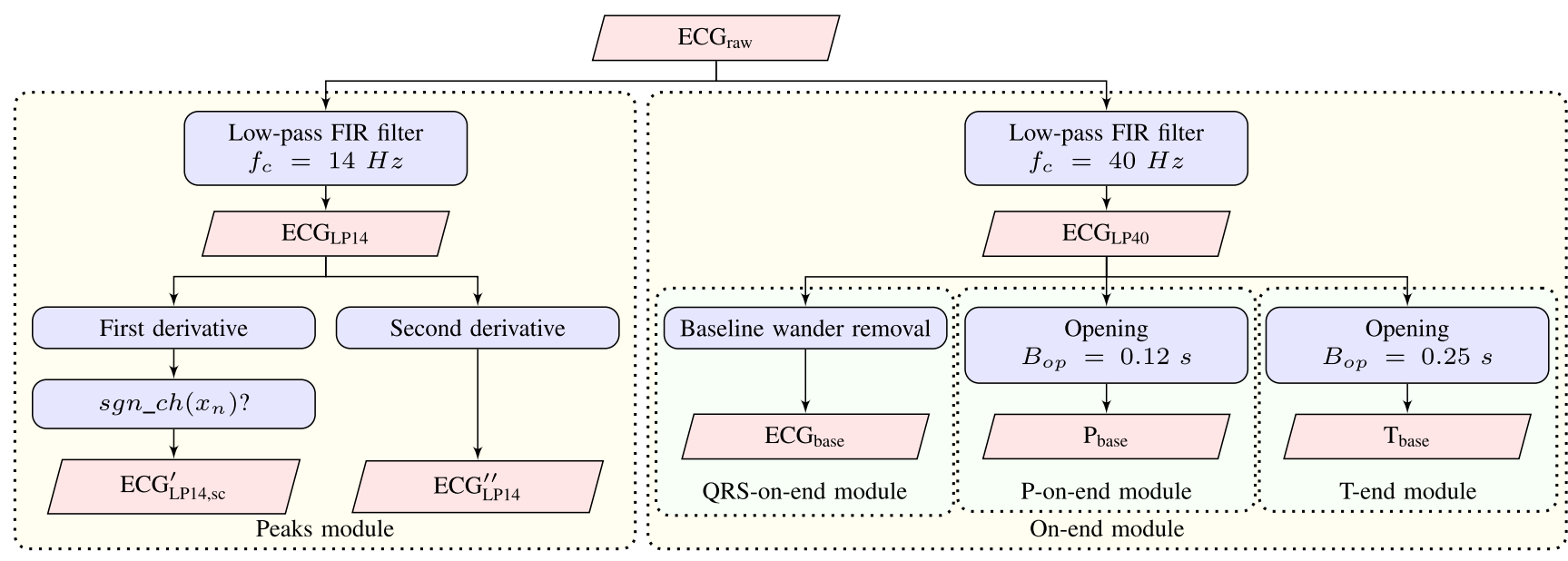

Fig. 3. Flowchart of the proposed modular ECG signal processing.

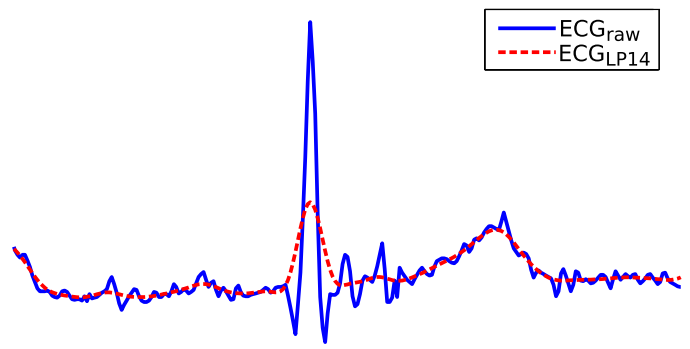

Fig. 4. Comparison between $E C G_{\text {raw }}$ signal and $E C G_{L P 14}$.

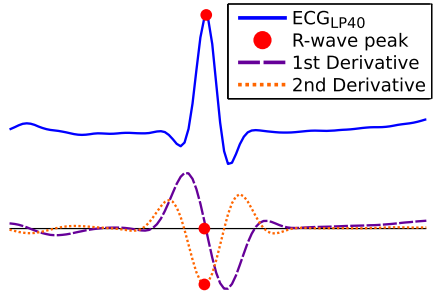

(a)

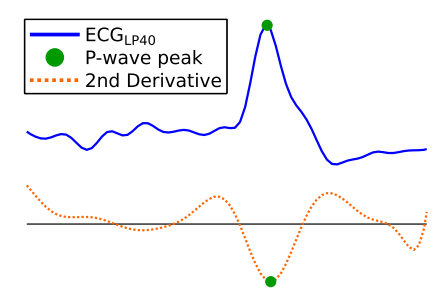

(b)
Fig. 5. (a) Positive QRS peak (R peak): the second derivative of $E C G_{L P 14}$ is negative and the first derivative crosses the zero line. (b) Positive P peak: the second derivative of $E C G_{L P 14}$ has a local min between 100 and 200 ms before a QRS complex. (a) QRS-peak (R wave). (b) $P$ peak.

$200 \mathrm{~ms}$ before a QRS peak, never exceeding half the time between the current QRS peak and the previous one, in order to avoid confusing a wave of the previous beat as the current $\mathrm{P}$ wave.

For a candidate point to be considered as the peak of the $\mathrm{P}$ wave, the second derivative of $E C G_{L P 14}$ has to be a minimum/maximum in the search window (see Fig. 5(b)) and exceed a certain threshold, $P_{t h}=P_{\text {factor }} \cdot A v g_{Q R S}$, similarly to the procedure used for QRS peak detection. For normal or positive $\mathrm{P}$ waves, the minimum of the second derivative is greater (in absolute value) than the maximum and, for abnormal or negative $\mathrm{P}$ waves, the maximum is greater (in absolute value) than the minimum. In this case, the threshold used to avoid false detections is chosen empirically as $1 \%$ of the second derivative

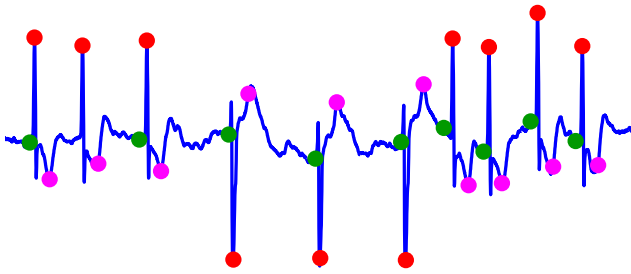

Fig. 6. A delineated ECG excerpt in which inverted QRS complexes and T waves are found (sel50 in QT Database).

of $E C G_{L P 14}$ in the QRS peak position $\left(P_{\text {factor }}=0.01\right)$, and will be justified in the next section. If this condition is not met, the $\mathrm{P}$ wave is not annotated.

The last characteristic wave of a heart beat is the $\mathrm{T}$ wave. The QT interval has to be shorter than $425 \mathrm{~ms}$ and the $\mathrm{T}$ wave duration is from $125 \mathrm{~ms}$ to $200 \mathrm{~ms}$ for a normal T wave [26]. Under these circumstances, the delineation algorithm searches the peak of the T wave in the time interval between $200 \mathrm{~ms}$ and $400 \mathrm{~ms}$ after a QRS complex, and never exceeding half the time between the current QRS peak and the next one.

The procedure to detect a $\mathrm{T}$ wave is exactly the same as in the $\mathrm{P}$ wave detection explained before. Also, the threshold used is $T_{t h}=T_{\text {factor }} \cdot A v g_{Q R S}$, with $T_{\text {factor }}=0.01$ and will be discussed in the next section.

\section{B. On-End Module}

In parallel to the Peaks module, the On-end module can be enabled for detecting onsets and ends of the previously detected ECG waves. A less aggressive low-pass FIR filter is applied to $E C G_{\text {raw }}$ with a cut-off frequency of $40 \mathrm{~Hz}$ (signal $E C G_{L P 40}$ ). This FIR filter cleans high frequency noise preserving the ECG waveform, and therefore $E C G_{L P 40}$ is suitable to detect onsets and ends of ECG waves.

1) QRS-on-End Module: To be able to detect ECG waves onsets and ends, the ECG baseline wander has to be removed from $E C G_{L P 40}$. In order to do so, $E C G_{L P 40}$ is fed to a highpass filter producing the ECG baseline signal ( $\left.E C G_{\text {ba se }}\right)$. Methods using FIR filters [27] or morphological filters [28] can be 


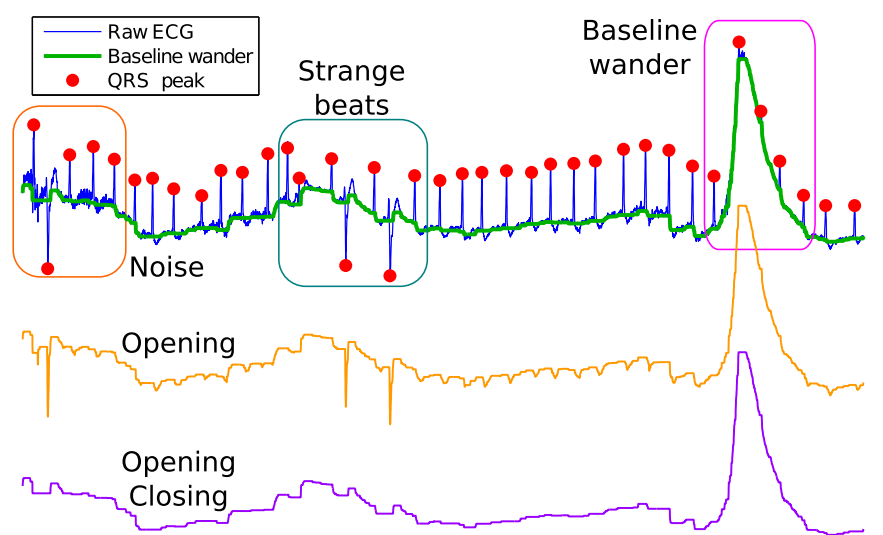

Fig. 7. ECG excerpt that contains noise, strange beats and baseline wander. Opening removes peaks and closing (after opening) removes valleys. The latter (purple) is $E C G_{\text {base }}$, the baseline wander shown above in green.

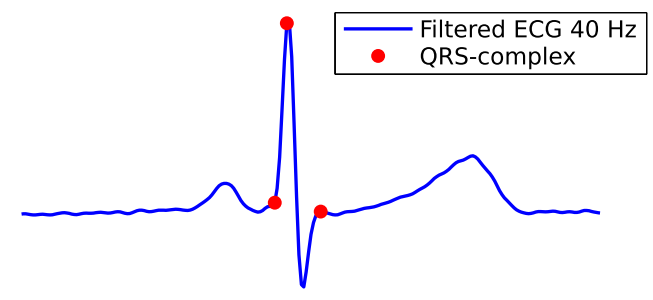

Fig. 8. The $Q R S$ complex presents an absent $Q$ wave.

found in other studies among most techniques to remove the ECG baseline wander. In this paper, morphological filtering described in [28] is used.

This filter consist of two basic morphological operations: opening, $f \circ B_{o p}=f \ominus B_{o p} \oplus B_{o p}$, (that removes peaks from the signal) and closing, $f \bullet B_{c l}=f \oplus B_{c l} \ominus B_{c l}$, (removes valleys), where $f$ is the signal to be filtered, $B$ denotes a horizontal line segment of zero amplitude, and $\ominus$ and $\oplus$ correspond with the erosion and dilation operands respectively. The length of $B_{o p}$ is chosen as $0.2 \mathrm{~s}$ because this value is greater than the width of the characteristic waves of the ECG signal (P, Q, R, S and $\mathrm{T}$ wave, individually). The length of $B_{c l}$ should be longer than the length of $B_{o p}$, typically it is selected to be 1.5 times the length of $B_{o p}$, therefore $0.3 \mathrm{~s}$ is chosen. Each operation introduces a delay that is equal to half of the window length used, thus the morphological filter has a total delay of $0.5 \mathrm{~s}$.

This type of filter presents a lower computational cost than other techniques since it only requires to find the largest or smallest value in an array of B values. An example of $E C G_{b a s e}$ (Baseline wander) along with opening and closing operations is shown in Fig. 7.

In the vicinity of a QRS peak, the ECG baseline wander $\left(E C G_{\text {base }}\right)$ is used to detect its onset and end. Supposing a positive QRS complex, the QRS peak is an R peak. Theoretically, the crossing of $E C G_{L P 40}$ with $E C G_{b a s e}$ determines two characteristic points: the previous crossing would be the onset of the $\mathrm{R}$ wave and the later crossing would be the end of the $\mathrm{R}$ wave.

However, the $E C G_{L P 40}$ might not cross $E C G_{\text {base }}$ near the $\mathrm{R}$ wave, especially if the $\mathrm{Q}$ or $\mathrm{S}$ waves are absent (Fig. 8). To prevent that the algorithm fails to detect the onset and end properly, these points are selected as $5 \%$ of the $\mathrm{R}$ wave amplitude instead of the true baseline crossings, where the amplitude is defined as the difference between $E C G_{L P 40}$ and $E C G_{b a s e}$ in the $\mathrm{R}$ peak position.

Once the onset of the $\mathrm{R}$ wave is found, a $\mathrm{Q}$ wave may be detected before the onset of the $\mathrm{R}$ wave. If the $\mathrm{Q}$ wave is present, it is smaller than the $\mathrm{R}$ wave and its duration does not exceed 20/25 ms in a normal Q wave [29]. To consider the existence of a $\mathrm{Q}$ wave, three rules have to be met:

1) The wave is found before an $R$ wave and is negative.

2) Its duration is less than $100 \mathrm{~ms}$.

3) Its amplitude is greater than $5 \%$ of the $\mathrm{R}$ wave amplitude. This value is chosen empirically and detections below this value are considered as variations due to noise.

Finally, if a $\mathrm{Q}$ wave is detected, the first crossing of $E C G_{L P 40}$ with $E C G_{b a s e}$ determines the onset of the $\mathrm{Q}$ wave. That point is also annotated as the onset of the QRS complex. Nevertheless, if the algorithm does not detect the existence of a $\mathrm{Q}$ wave, the onset of the $\mathrm{R}$ wave is annotated as the onset of the QRS complex. In order to improve the final positioning of the QRS complex onset, the algorithm searches for a local maximum of $E C G_{L P 40}$ in the previous $20 \mathrm{~ms}$. If such maximum exists, the onset of the QRS complex is updated to that new position.

If an $\mathrm{R}$ wave is detected, a $\mathrm{S}$ wave may follow it. The requirements of considering $\mathrm{S}$ waves and the treatment are the same as for $\mathrm{Q}$ waves. The only difference is that the search occurs (in time) after $\mathrm{R}$ peaks instead of before. If a $\mathrm{S}$ wave is annotated, the end of the $S$ wave is also the end of the QRS complex. Otherwise, the end of the $\mathrm{R}$ wave is the end of the QRS complex.

In case of negative QRS complexes, the detection is analogous, but the QRS peak corresponds to an $\mathrm{S}$ wave and the algorithm looks for positive deflections ( $\mathrm{R}$ waves) before and after that $\mathrm{S}$ wave.

2) P-on-End Module: To detect the onset and end of the $\mathrm{P}$ wave, the crossings of $E C G_{L P 40}$ with the baseline have to be found (to the left and right of the peak, respectively). For this purpose, the $P_{b a s e}$ signal (see Fig. 3) is used. This signal is obtained by applying the morphological operation defined previously as opening (II-B1) to $E C G_{L P 40}$, which removes the peaks of the signal, in the same way as in $E C G_{b a s e}$. However, the length of the segment $B_{o p}$ in this case differs. Since a normal $\mathrm{P}$ wave has a maximum length of $120 \mathrm{~ms}$, the length of $B_{o p}$ must be in agreement with this physiological property. Choosing $B_{o p}=120 \mathrm{~ms}$, opening removes peaks whose width is less than this time and makes a good approximation to the baseline of a $\mathrm{P}$ wave.

As well as for a QRS complex, the onset and end of a $\mathrm{P}$ wave are, theoretically, the crossings of $E C G_{L P 40}$ with $P_{b a s e}$. However, it may be possible that these crossings do not take place near the peak of the $\mathrm{P}$ wave. For this reason, the onset and end are defined as the first local minima (to the left and right of the peak, respectively) found in $E C G_{L P 40}$ for which the difference between $E C G_{L P 40}$ and $P_{b a s e}$ is between $50 \%$ and $2.5 \%$ of the amplitude of the $\mathrm{P}$ wave, defined as the difference between $E C G_{L P 40}$ and $P_{b a s e}$ in the P peak position.

3) T-End Module: As in the detection of the onset and end of the $\mathrm{P}$ wave, the baseline of the $\mathrm{T}$ wave $\left(T_{\text {base }}\right)$ is needed (see 


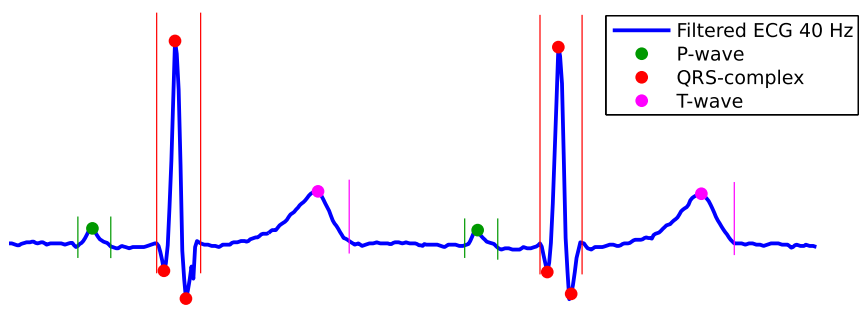

Fig. 9. A delineated ECG excerpt (se/16539 in QT Database).

Fig. 3). This signal is computed using the opening operation (IIB1) with a $B_{o p}=200 \mathrm{~ms}$, as a normal T wave must not exceed $200 \mathrm{~ms}$. The crossing of $E C G_{L P 40}$ with $T_{\text {base }}$ to the right of the peak of the $\mathrm{T}$ wave should determine the end. However, as in $\mathrm{P}$ waves, this point has been redefined as the first local minima found in $E C G_{L P 40}$ for which the difference between $E C G_{L P 40}$ and $T_{\text {base }}$ is between $50 \%$ and $2.5 \%$ of the $\mathrm{T}$ wave amplitude, in order to obtain better results. The algorithm is able to detect both types of $\mathrm{T}$ waves, normal and abnormal, although biphasic $\mathrm{T}$ waves may not be accurately detected.

A complete result of the ECG delineation described is shown in Fig. 9. This excerpt corresponds to the sel16539 record obtained from the QT database [30].

\section{Validation of the Algorithm}

The described algorithm has been validated using the QT database [30] and managed from MATLAB ${ }^{\& \&}$ using the WFDB toolbox [31], [32]. This database consists of 105 15-minute excerpts of two-lead ECG recordings, sampled at $250 \mathrm{~Hz}$. For each record, between 30 and 70 beats (including P waves, QRS complexes and $\mathrm{T}$ waves) are manually annotated by cardiologists.

The proposed algorithm works on a single-lead signal, thus the comparison is performed selecting each annotated sample in the two channels of each database record and searching if the algorithm finds the same type of annotation in the vicinity $(150 \mathrm{~ms})$ of that manually annotated sample. This time interval has been chosen as indicated by the standard [33], in which The Association for the Advancement of Medical Instrumentation (AAMI) describes the metrics that should be used for algorithm validation. This time interval will be called "tolerance" in the rest of the paper.

If the algorithm correctly detects a point, a true positive $(T P)$ detection is counted and the error is calculated as the time interval between the manually-annotated point in the database and automatic detection carried out by the algorithm. The algorithm is executed separately in each lead of the QT database, and for each point, the one that presents the smallest error is chosen. If the algorithm does not detect a point, a false negative $(F N)$ detection is counted. Once every detected point by the algorithm is compared with the corresponding manual annotations of the database, any point that has not been related to the database is considered as a false positive $(F P)$. Based on this, 4 different metrics are defined for the evaluation of the algorithm: mean error $(m)$, standard deviation of the mean error $(\sigma)$, sensitivity $(S e(\%)=T P /(T P+F N))$ and positive predictive value $(P P V(\%)=T P /(T P+F P))$. This validation procedure has been chosen to perform a fair comparison with previous works, such as [5], [21], [34] that will be presented in the next section.

Table I shows the results obtained by the algorithm delineating the QT database using the metric defined by the AAMI with order-40 FIR filters for $E C G_{L P 14}$ and $E C G_{L P 40}$. The results show that the sensitivity for the peaks of the P, QRS and $\mathrm{T}$ waves is higher than $98 \%$ and the standard deviation of the mean error for each delineated point is below the tolerances of the Common Standards for Electrocardiography (CSE) committee [35] ( $s<2 \sigma_{C S E}$, known as "loose criterion"), except for the onset of the $\mathrm{P}$ wave, for which this value is $3 \mathrm{~ms}$ above. The end of the $\mathrm{T}$ wave has the highest mean error but below the tolerance, the proper delineation of this point is a well-known challenge, even for cardiologists and different studies have been developed discussing this issue [36].

\section{Adjusting the Algorithm Parameters}

As aforementioned, there are some parameters that have to be tuned off-line to optimize the results (FIR filters parameters: cut-off frequency and order) or change the behavior of the wave peak detection $\left(R_{\text {factor }}, P_{\text {factor }}\right.$ and $\left.T_{\text {factor }}\right)$. In order to study how these parameters affect the performance, the metrics introduced before have been studied during the delineation of the QT database.

The cut-off frequency of the FIR filter used in the algorithm for the Peaks module to calculate the first and second derivatives and detect the wave peaks has been selected using Fig. 10(a). As it can be seen, if the frequency is lower than $10 \mathrm{~Hz}$, the sensitivity is high but the PPV is low. As the frequency increases, the sensitivity starts decreasing after $15 \mathrm{~Hz}$ while the PPV always increases. The best results involve a good trade-off between these two values taking into account the standard deviation, thus the best final choice for the cut-off frequency is $14 \mathrm{~Hz}$ for which the sensitivity and the PPV are the highest, while keeping a good value for the standard deviation.

Filter orders from 10 to 40 for $E C G_{L P 14}$ and $E C G_{L P 40}$ present the best results: order 40 shows a better accuracy in delineation but also higher CPU consumption than order 10 .

Another parameter, which can be used to control the detection of QRS peaks, is $R_{\text {factor }}$, whose range is from 0 to 1 . As shown in Fig. 10(b), if this factor is high (about 0.7 or 0.8 ) false negative detections increase while false positive detections decrease, since less QRS peaks are detected. This situation involves worse sensitivity but better PPV. On the other hand, a low $R_{\text {factor }}$ (about 0.1 or 0.2 ) leads to higher sensitivity and lower PPV. Finally, analyzing the results, the $R_{\text {factor }}$ was selected to be 0.33 , since the sensitivity and the PPV are balanced with a low standard deviation of the mean error. This parameter can be used to fine-tune the algorithm according to the use case, depending on whether the system wants to maximize the sensitivity or the positive predictivity, and therefore the ratio or false positive and false negative detections.

The same reasoning can be applied to choose $P_{\text {factor }}$ and $T_{\text {factor }}$, which detect the peaks of the P (Fig. 10(c)) and T waves (Fig. 10(d)) respectively. In this paper, both factors have 
TABLE I

Results for the Delineation in High-Accuracy Mode of the QT Database According to the Standards Recommended by the AAMI $($ TOLERANCE $=150 \mathrm{MS})$

\begin{tabular}{|c|c|c|c|c|c|c|c|c|}
\hline Beats & 3194 & 3194 & 3194 & 3623 & 3623 & 3623 & 3542 & 3542 \\
\hline$F N$ & 102 & 58 & 9 & 0 & 0 & 13 & 36 & 135 \\
\hline$F P$ & 232 & 188 & 140 & 18 & 18 & 28 & 73 & 186 \\
\hline $\mathrm{Se}(\%)$ & 96.81 & 98.18 & 99.72 & 100.00 & 100.00 & 99.64 & 98.98 & 96.19 \\
\hline $2 \sigma_{C S E}(\mathrm{~ms})$ & 10.2 & - & 12.7 & 6.5 & - & 11.6 & - & 30.6 \\
\hline
\end{tabular}

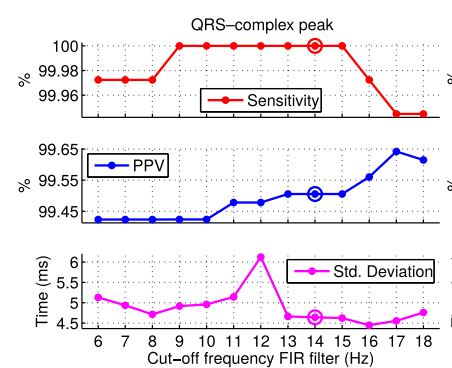

(a)

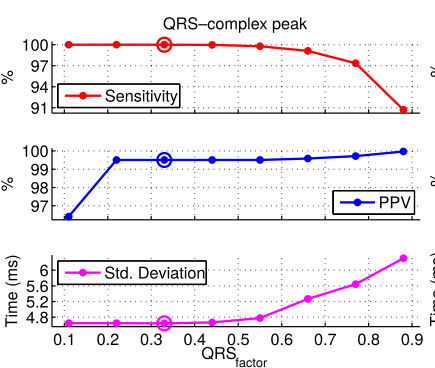

(b)

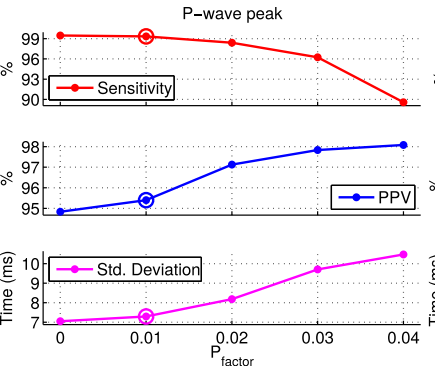

(c)

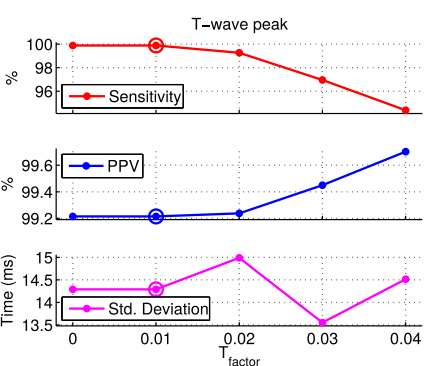

(d)

Fig. 10. Sensitivity, positive predictive value and standard deviation of the mean error for (a) QRS peaks versus FIR filter cutoff frequency of the Peaks module and (b)-(d) peaks of QRS, P and T waves versus second derivative factor used as thresholds. (a) QRS peak. (b) QRS peak. (c) P peak. (d) T peak.

been chosen as 0.01 which allow a high sensitivity and a good value for the standard deviation.

\section{E. Computational Cost Versus Accuracy Tradeoff}

As previously said, the algorithm presents the best results with a sampling rate of $250 \mathrm{~Hz}$ and a 40-order FIR filters, Table I. In this mode, which will be denoted as High-accuracy mode in the following sections, the proposed algorithm presents similar results in terms of accuracy and CPU consumption as other algorithms, such as [21]. The main advantage of the proposed technique with respect to previous works is its ability to operate in different modes, that can be selected dynamically at run time, depending on which ECG fiducial points have to be detected and how accurate the detection must be, and therefore drastically reducing the CPU usage.

Table II show possible modes of the delineation algorithm denoted as: High-accuracy, Standard, Low-power and Ultralow-power modes. In the Standard modes and High-accuracy, the algorithm is able to just detect the beats (QRS peaks and HR) or to perform a full delineation (QRS, $\mathrm{P}$ and $\mathrm{T}$ waves), whereas in Low-power and Ultra-low-power modes, the sampling rate is reduced to $50 \mathrm{~Hz}$ and only beats are detected, as will be explained later.

In High-accuracy mode, the main contribution of the algorithm to the CPU usage consists of the ECG filtering, as it will be shown in Section III. During delineation, $50 \%$ of the CPU time is dedicated to implement the FIR filters, $40 \%$ to the morphological filtering and only $10 \%$ to the analysis of the fil- tered signals, and therefore, reducing the computational cost due to filtering has a great impact on the overall CPU usage, although will degrade the delineation accuracy. This reduction can be achieved by disabling the detection of some fiducial points or by reducing the filter complexity. The difference between High-accuracy and Standard modes is that the latter uses 10-order FIR filters and, as a result, has less CPU usage with a slight reduction in accuracy.

If the amount of samples per second to be filtered is reduced (Low-power mode), less CPU will be used to filter the signal. The QRS peaks of the QT database have been delineated at $50 \mathrm{~Hz}$ (with a $Q R S_{\text {factor }}=0.66$ ) allowing good QRS peak detection and CPU reduction, Table II, and even the possibility of switching on and off the ADC between samples, e.g. the ADS1292 [37] has a $10 \mathrm{~ms}$ wake-up time. Unfortunately, $50 \mathrm{~Hz}$ is not suitable for accurate $\mathrm{P}$ and $\mathrm{T}$ wave delineation.

But one step further is possible. If the heart rate (HR) is regular, we can estimate when the next beat will occur, switching off the ADC not only between samples but also between QRS peaks. This is how Ultra-low-power mode works. In this mode, the ADC is switched on 11 samples $(220 \mathrm{~ms})$ before the next expected QRS peak (5 samples to feed the 10-order FIR filter, 1 to compute the first derivative and 5 more samples to address possible HR increments) and is switched off 6 samples after the QRS peak detection (the minimum number of required samples to process the signals and detect QRS peaks). This mode continues running as long as heart rate (HR) is regular. In this mode (Ultra-low-power mode), if the HR is not stable, the algorithm will change to Low-power mode, sampling continu- 
TABLE II

Different Operating Modes of the Proposed Algorithm

\begin{tabular}{|c|c|c|c|c|}
\hline Mode & High-accuracy & Standard & Low-power & Ultra-low-power \\
\hline Features & $\begin{array}{c}\text { High frequency } \\
\text { High-order FIR filters }\end{array}$ & $\begin{array}{c}\text { High frequency } \\
\text { Low-order FIR filters }\end{array}$ & $\begin{array}{c}\text { Low frequency } \\
\text { Continuous sampling }\end{array}$ & $\begin{array}{c}\text { Low frequency } \\
\text { Discontinuous sampling }\end{array}$ \\
\hline Detectable waves & QRS, P, T peaks, onsets, ends & QRS, P, T peaks, onsets, ends & QRS peak & QRS peak \\
\hline Sampling rate $(\mathrm{Hz})$ & 250 & 250 & 50 & 50 \\
\hline Filter order & 40 & 10 & 10 & 10 \\
\hline Computational cost & Medium-high & Medium & Low & Ultra-low \\
\hline Accuracy & Ultra-high & High & Medium-high & Medium \\
\hline \multicolumn{5}{|c|}{ QRS peak delineation $(Q T D B)$} \\
\hline$S e-P P V(\%)$ & $100-99.51$ & $99.50-99.78$ & $97.27-98.27$ & $95.83-98.78$ \\
\hline$m \pm \sigma(m s)$ & $-6.0 \pm 4.6$ & $-5.5 \pm 6.6$ & $20.47 \pm 24.46$ & $22.15 \pm 26.45$ \\
\hline
\end{tabular}

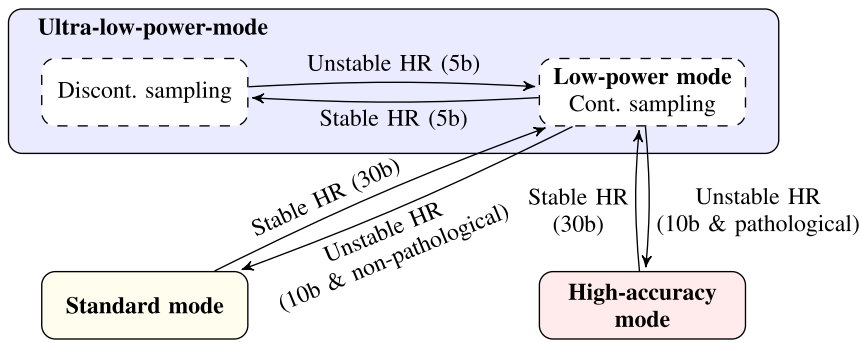

Fig. 11. State diagram of the Adaptive mode.

ously. The results for Ultra-low-power mode are presented in Table II.

Fig. 11 shows a possible system configuration combining the previously described modes, denoted as Adaptive mode. The transitions are based on the stability of the HR and also consider if the patient has already been diagnosed with a pathology. We define a regular HR when the current HR (the last HR value from the last 2 QRS peaks) is between $75 \%$ and $125 \%$ of the average HR during the last $n$ beats. In Ultra-low-power mode, transitions between Discontinuous sampling and Continuous sampling occur based on the stability of the HR during the last 5 beats. The transition from Ultra-low-power mode to Standard mode or High-accuracy mode takes place based on the same stability condition of the HR, but using the last 10 beats, moving towards High-accuracy mode if the patient has previous known pathologies or towards Standard mode if not. The system returns to Ultra-low-power mode when the HR is stable during the last 30 beats. This scenario is a way to illustrate that the algorithm can change the mode in real time, and our selection of the values is only a possible practical example.

The delineation algorithm can automatically switch between modes in real time thanks to: (1) all the filters are easily adaptable to the sampling rate and (2) the modularity of the algorithm's schema.

1) The Delineation Algorithm is Easily Adaptable to the Sampling Rate: The parts of the algorithm that are frequencydependent are just the FIR filters and the morphological filters. This implies that these parts have to be modified if the ECG sampling rate changes, however, the adaptations are straightforward. The FIR filters coefficients have to be adjusted to maintain the filter cut-off frequency according to the Nyquist frequency and the morphological operations have to modify their window lengths since they are time-domain defined, but the algorithm remains unmodified.

2) The Delineation Algorithm Is Modular: This modularity (Fig. 3) makes this algorithm more flexible and adaptive in contrast to other techniques such as wavelets [5], [21]. In case of abnormal cardiac episodes, a complete delineation is required. However, there can be patients for whom we could only want information about parameters, e.g. HR, for which a full delineation would not be necessary. When a full delineation is required, and in order to extract all the possible information about the event (peaks, onsets and ends for P, T waves and QRS complexes) in real time, the High-accuracy and Standard modes can be selected. When a full delineation is not required, the Low-power and Ultra-low-power modes can be used. Thanks to the modularity of the proposed approach, the algorithm can perform a selected delineation where the system decides which ECG waves have to be delineated and which fiducial points are detected. The rest of the time, a reduced delineation (i.e. QRS peak detection using Low/Ultra-low power power mode) can be executed, reducing the computational load of the delineation and, therefore, using energy more efficiently.

\section{RESULtS}

In this section the delineation algorithm is compared with other techniques using the QT database. The MIT-BIH Arrhythmia database is delineated to show how the algorithm behaves with another real ECG database, with noise and different sampling rate. Also, real tests have been performed with a Shimmer node during real live situations, sampled at $1 \mathrm{kHz}$ and $5 \mathrm{kHz}$. The obtained results were satisfactory but not included in the paper because they were not validated by a cardiologist. Finally, the results of a profiling of the delineation algorithm are presented, using a simulator of the Shimmer platform, in terms of computational burden, memory footprint and the estimated power consumption and an evaluation of the complexity of the algorithm.

\section{A. Comparison With Other Methods}

Table III shows the results obtained by the proposed and other state-of-the-art techniques, such as wavelets [5], [21], [34] or phasor transform [7], delineating the QT database. It should be noted that, since the time window to match the automatic and 
TABLE III

COMPARISON FOR DIFFERENT DELINEATION ALGORITHMS ON THE QT DATABASE

\begin{tabular}{|c|c|c|c|c|c|c|c|c|}
\hline & & $P$-onset & P-peak & $P$-end & $Q R S$-onset & $Q R S$-end & T-peak & T-end \\
\hline $\begin{array}{l}\text { This work High-accuracy mode } \\
\text { (tol = } 200 \mathrm{~ms} \text { ) }\end{array}$ & $\begin{array}{c}\operatorname{Se}(\%) \\
P P V(\%) \\
m \pm \sigma(m s)\end{array}$ & $\begin{array}{c}98.22 \\
94.23 \\
22.3 \pm 14.0 \\
\end{array}$ & $\begin{array}{c}99.34 \\
95.40 \\
13.5 \pm 7.3\end{array}$ & $\begin{array}{c}99.87 \\
95.88 \\
-0.7 \pm 9.5 \\
\end{array}$ & $\begin{array}{c}100.00 \\
99.51 \\
7.0 \pm 4.3 \\
\end{array}$ & $\begin{array}{c}99.97 \\
99.48 \\
-5.0 \pm 9.9 \\
\end{array}$ & $\begin{array}{c}99.89 \\
99.21 \\
8.4 \pm 14.3 \\
\end{array}$ & $\begin{array}{c}97.49 \\
96.67 \\
-11.7 \pm 15.0 \\
\end{array}$ \\
\hline $\begin{array}{l}\text { This work Standard mode } \\
\text { (tol }=200 \mathrm{~ms} \text { ) }\end{array}$ & $\begin{array}{c}\operatorname{Se}(\%) \\
P P V(\%) \\
m \pm \sigma(m s)\end{array}$ & $\begin{array}{c}98.12 \\
94.26 \\
23.9 \pm 19.5 \\
\end{array}$ & $\begin{array}{c}99.15 \\
95.11 \\
13.8 \pm 8.8\end{array}$ & $\begin{array}{c}99.87 \\
96.03 \\
-1.9 \pm 10.4 \\
\end{array}$ & $\begin{array}{c}99.50 \\
99.78 \\
6.4 \pm 5.5 \\
\end{array}$ & $\begin{array}{c}99.50 \\
99.78 \\
-5.2 \pm 10.8 \\
\end{array}$ & $\begin{array}{c}99.41 \\
98.96 \\
9.0 \pm 15.4 \\
\end{array}$ & $\begin{array}{c}96.98 \\
95.98 \\
-12.9 \pm 18.6 \\
\end{array}$ \\
\hline Rincón et al. [21] & $\begin{array}{c}\mathrm{Se}(\%) \\
\mathrm{PPV}(\%) \\
m \pm \sigma(m s)\end{array}$ & $\begin{array}{c}99.87 \\
91.98 \\
8.6 \pm 11.2\end{array}$ & $\begin{array}{c}99.87 \\
92.46 \\
10.1 \pm 8.9\end{array}$ & $\begin{array}{c}99.91 \\
91.70 \\
0.9 \pm 10.1\end{array}$ & $\begin{array}{c}99.97 \\
98.61 \\
3.4 \pm 7.0\end{array}$ & $\begin{array}{c}99.97 \\
98.72 \\
3.5 \pm 8.3\end{array}$ & $\begin{array}{c}99.97 \\
98.91 \\
3.7 \pm 13.0\end{array}$ & $\begin{array}{c}99.97 \\
98.50 \\
-2.4 \pm 16.9\end{array}$ \\
\hline Di Marco et al. [34] & $\begin{array}{c}\operatorname{Se}(\%) \\
P P V(\%) \\
m \pm \sigma(m s)\end{array}$ & $\begin{array}{c}98.15 \\
91.00 \\
-4.5 \pm 13.4\end{array}$ & $\begin{array}{c}98.15 \\
91.00 \\
-4.7 \pm 9.7\end{array}$ & $\begin{array}{c}98.15 \\
91.00 \\
-2.5 \pm 13.0\end{array}$ & $\begin{array}{c}100.00 \\
- \\
5.1 \pm 7.2\end{array}$ & $\begin{array}{c}100.00 \\
- \\
0.9 \pm 8.7\end{array}$ & $\begin{array}{c}99.72 \\
97.76 \\
-0.3 \pm 12.8\end{array}$ & $\begin{array}{c}99.77 \\
97.76 \\
1.3 \pm 18.6\end{array}$ \\
\hline Marínez et al. [5] & $\begin{array}{c}\operatorname{Se}(\%) \\
P P V(\%) \\
m \pm \sigma(m s)\end{array}$ & $\begin{array}{c}98.87 \\
91.03 \\
2.0 \pm 14.8\end{array}$ & $\begin{array}{c}98.87 \\
91.03 \\
3.6 \pm 13.2\end{array}$ & $\begin{array}{c}98.75 \\
91.03 \\
1.9 \pm 12.8\end{array}$ & $\begin{array}{c}99.97 \\
- \\
4.6 \pm 7.7\end{array}$ & $\begin{array}{c}99.97 \\
- \\
0.8 \pm 8.7\end{array}$ & $\begin{array}{c}99.97 \\
97.79 \\
0.2 \pm 13.9\end{array}$ & $\begin{array}{c}99.77 \\
97.79 \\
-1.6 \pm 18.1\end{array}$ \\
\hline Marínez et al. [7] & $\begin{array}{c}\operatorname{Se}(\%) \\
P P V(\%) \\
m \pm \sigma(m s)\end{array}$ & $\begin{array}{c}98.65 \\
- \\
2.6 \pm 14.5\end{array}$ & $\begin{array}{c}98.65 \\
- \\
32 \pm 25.7\end{array}$ & $\begin{array}{c}98.65 \\
- \\
0.7 \pm 14.7\end{array}$ & $\begin{array}{c}99.85 \\
- \\
-0.2 \pm 7.2\end{array}$ & $\begin{array}{c}99.85 \\
- \\
2.5 \pm 8.9\end{array}$ & $\begin{array}{c}99.20 \\
- \\
5.3 \pm 12.9\end{array}$ & $\begin{array}{c}99.20 \\
- \\
5.8 \pm 22.7\end{array}$ \\
\hline$\overline{2 \sigma_{C S E}(m s)}$ & & 10.2 & - & 12.7 & 6.5 & 11.6 & - & 30.6 \\
\hline
\end{tabular}

manual annotations is different for each work, the comparisons are not perfectly accurate. In [34], the length of the time window is $150 \mathrm{~ms}$, in [21] is $320 \mathrm{~ms}$ and in [5] and [7] this information is not reported. If the interval is larger, the sensitivity, PPV and standard deviation is higher. To be fair and closer to other reported results, the chosen time window in this comparison table is $200 \mathrm{~ms}$.

Table III reports the results for the algorithm modes that works at $250 \mathrm{~Hz}$ : the High-accuracy and the Standard mode. The results show that the former presents better accuracy than latter but, for both modes, the sensitivity, PPV and standard deviation are within the CSE committee tolerances, except for the aforementioned $\mathrm{P}$ wave onset.

As it can be seen, the values of the sensitivity are very similar to the others, despite the fact that this value is directly related to the chosen time interval to accept or discard a delineated point as valid. In particular, the PPVs obtained for this work are higher than for other techniques for the $\mathrm{P}$ wave and lower for the end of the $\mathrm{T}$ wave, which demonstrates the difficulty to delineate this point. For the standard deviation of the mean error, which is the most important parameter since it illustrates the dispersion of the errors, the results are in agreement with the CSE and show that the proposed algorithm can delineate ECG fiducial points like the onset and the end of the QRS complex, or the peak and the end of the $\mathrm{P}$ wave, with less dispersion than other more complex techniques.

\section{B. MIT-BIH Arrhythmia Database}

Additionally to the delineation of the QT database, and with the aim of evaluating the performance of the algorithm with other real ECG signals, the MIT-BIH Arrhythmia database [31], [38] has been delineated. This database consists of 48 30-minutes excerpts of 2-channel ambulatory ECG recordings with annotated beats and sampled at $360 \mathrm{~Hz}$.
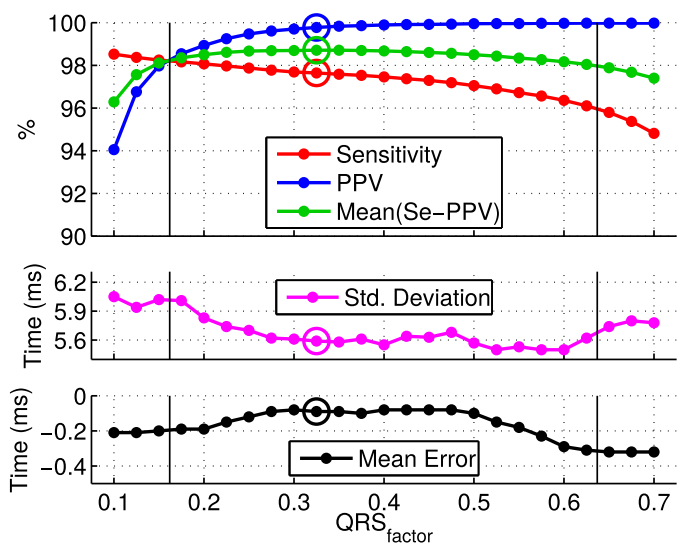

Fig. 12. Sensitivity, PPV, mean error and std. dev. of the mean error for different values of $Q R S_{\text {factor }}$ at delineating the MIT-BIH Arrhythmia DB. The best range is between 0.15 and 0.65 , and 0.33 is the optimal value.

All the registers (a total of 112646 beats) have been delineated by the algorithm and the only change made to the parameters of the algorithm to delineate the MIT-BIH database instead of the QT database is the numerical values of the coefficients of the 2 FIR filters, to adjust their cutoff frequencies from the sampling rate of $250 \mathrm{~Hz}$ to $360 \mathrm{~Hz}$, according to the Nyquist frequency. The rest of the algorithm parameters remain the same.

With the goal of showing the performance of the algorithm in beats detection, and justifying the selection of $Q R S_{\text {factor }}$ as 0.33 (parameter obtained for the QT database delineation) as the best value for this threshold, the MIT-BIH database has been delineated for a wide range of $Q R S_{\text {factor }}$ (note that $Q R S_{\text {factor }}$ has meaning from 0 to 1 ). The results (in terms of sensitivity, PPV, mean error and standard deviation of the mean error) are shown in Fig. 12. 
TABLE IV

Results of Beat Detection for the Register 118 (MIT-BIH NoIse STRESS TEST DB) WITH DIFFERENT SNRS

\begin{tabular}{lcccccc}
\hline \hline $\operatorname{SNR}(d B)$ & 24 & 18 & 12 & 6 & 0 & -6 \\
\hline $\operatorname{Se}(\%)$ & 99.0 & 99.0 & 99.1 & 99.2 & 99.2 & 98.2 \\
$\operatorname{PPV}(\%)$ & 99.9 & 99.9 & 99.4 & 91.7 & 79.8 & 76.6 \\
\hline \hline
\end{tabular}

As can be seen, any value between 0.15 and 0.65 for $Q R S_{\text {factor }}$ presents similar and stable results. Below 0.15, PPV decreases considerably because more false positives are detected and, on the other hand, values higher than 0.6 decrease the sensitivity (there are more false negatives). As for the QT database delineation, 0.33 is justified as an optimal value for MIT-BIH database as well (with almost $98 \%$ of sensitivity and almost $100 \%$ of PPV). Anyway, this threshold can be slightly modified within this range to try to get better sensitivity or better PPV in the detection.

1) Noise: In order to evaluate the performance of the algorithm under noisy conditions (different types and intensities of noises), the record 108 from MIT-BIH Noise Stress Test database [31], [39] has been delineated. This record contains 2301 annotated beats and the raw ECG mixed with different types of noises having different signal-to-noise ratios (SNR): from $24 \mathrm{~dB}$, where the ECG signal is clean, up to $-6 \mathrm{~dB}$, where the ECG is strongly affected by baseline wander, muscle artifact and electrode motion artifact.

Table IV shows the results of the algorithm in beat detection for the same ECG signal with different types of SNRs. As can be seen, for $12 \mathrm{~dB}$ or more, the algorithm maintains good beat detection rate, while for the same but more noisy signal (below $12 \mathrm{~dB}$ ), sensitivity remains constant but PPV decays, which means an increase of false positives due to noise.

\section{Estimated Number of Arithmetic Operations}

The algorithm is divided into three main tasks: FIR filtering, morphological filtering and the delineation of the ECG fiducial points. As shown in next section, approximately $90 \%$ of the computation time is devoted to FIR (50\%) and morphological (40\%) filtering. These two parts are the most computationally intensive and their number of operations gives roughly the complexity of the algorithm [40], since the delineation of the ECG points mainly requires comparisons and means the $10 \%$ of the computation time.

1) FIR Filtering: This part requires $8 N+2$ operations for each new ECG sample, where $N$ denotes the filter order, as explained bellow. When a new ECG sample arrives, $N$ rotations and $N$ index increments are needed for the input samples buffer; $N$ additions, $N / 2+1$ multiplications and $N-1$ index increments, for the convolution operation; and finally, 1 division (if the platform does not support the division, coefficient quantization can be used to avoid the division, as explained in next section). As the 2 FIR filters may share the input samples buffer, the number of operations required for the FIR filters for each new ECG sample is $8 N+2$. As an example, using a 40 order filter as in High-accuracy mode leads to 40 rotations, 158 index increments, 80 additions, 82 multiplications and 2 divisions (a total of 322 operations).

2) Morphological Filtering: This stage requires comparisons for finding the maximum or minimum per each new ECG sample of a total of 8 buffers (4 for $E C G_{b a s e}, 2$ for $P_{b a s e}$ and 2 for $T_{\text {base }}$ ), meaning 1 comparison or $L$ comparisons for each buffer (where $L$ is the length of the buffer defined in time domain) depending on each situation. A list of sub-maximum values, one per buffer, has been used to speed up the process and therefore in most cases a full window search is not necessary. This means that, if the $\mathrm{max} / \mathrm{min}$ is located in a buffer and does not change when a new ECG sample arrives, only 1 comparison is required. Occasionally, the $\max / \mathrm{min}$ has to be searched within the buffer because its position has been overwritten with a new ECG sample, so in the worst case, $L$ comparisons have to be made to find it again.

Assuming a sampling rate of $250 \mathrm{~Hz}$, there are: 2 opening buffers (length 49) and 2 closing buffers (length 75) for $E C G_{\text {base }} ; 2$ opening buffers (length 29) for $P_{\text {base }}$; and 2 opening buffers (length 63) for $T_{\text {base }}$. In the best case, that happens for most of the new samples, just 8 comparisons are needed because all $\mathrm{max} / \mathrm{min}$ are located (the position of the $\mathrm{max} / \mathrm{min}$ in each buffer is not overwritten when a new sample arrives, thus a new search is not necessary) and 4 extra comparisons for buffer indices (a total of 12 comparisons). In the worst case, less frequent, in which all $\mathrm{max} / \mathrm{min}$ have to be found in all the buffers, 432 comparisons are needed and 4 extra comparisons for buffer indices, and 432 increments of indices due to an iterator (a total of 868 operations).

\section{Real-Time Simulation}

The algorithm proposed has also been written in $\mathrm{C}$ language and simulated for a commercial WBSN device: the Shimmer ${ }^{\mathrm{TM}}$ 3 platform [41] to analyze the real computational burden and the memory footprint of the algorithm.

The Shimmer node is a battery powered wireless sensor node equipped with an ultra-low-power microcontroller, the TI MSP430F5437A (16-bit RISC architecture, operating at clock rate frequencies up to $25 \mathrm{MHz}, 16 \mathrm{kB}$ of RAM and $256 \mathrm{kB}$ of Flash memory), and the TI ADS1292, a complete low-power integrated analog front-end for ECG applications. The simulator used is the MSPSim [42], a Java-based instruction level emulator of the MSP430 series microprocessor. Since the simulator does not provide support for the Shimmer node, we have implemented modifications, based on Moteiv (Tmote Sky), to have two serial ports: USARTO as input port with the input ECG signal and USART1 as output port with the delineated points when a beat is delineated.

A 15-minute ECG excerpt of the QT Database has been used to profile the different modes. Table $\mathrm{V}$ shows the computational burden and the memory footprints used in the simulator of the Shimmer node for the different algorithm modes defined in Table II, and also the estimated power consumption and lifetime for the node, taking into account only the power consumption due to data acquisition by the ADS1298 and processing by 
TABLE V

Computational Burden, Memory Footprint, and Energy Parameters for Different Modes of the Algorithm in the Shimmer3 Node

\begin{tabular}{lcccccc}
\hline \hline Mode & Work [21] & High-accuracy & Standard & Low-power & Ultra-low-power & Adaptive \\
\hline Comp. burd. $(\%)$ & 6.8 & $8.5-3.3$ & $5.7-1.6$ & 0.4 & 0.2 & 1.3 \\
RAM $(k B)$ & 7.2 & $9.3-2.3$ & $9.3-2.2$ & 2.2 & 2.2 & 9.3 \\
Flash $(k B)$ & 15 & $9.1-4.8$ & $7.8-4.0$ & 4.0 & 4.0 & 9.1 \\
Power cons. $(\mu \mathrm{W})$ & 1050 & $1145-860$ & $990-765$ & 445 & 315 & 423 \\
Lifetime $($ weeks $)$ & 9.4 & $8.6-11.5$ & $10.0-13.0$ & 22.3 & 31.5 & 23.5 \\
Sampling Rate $(\mathrm{Hz})$ & 250 & $250-250$ & $250-250$ & 50 (Cont.) & 50 (Disc.) & $50($ Disc.)/250 \\
Delineation & Full & Full-QRS-peak & Full-QRS-peak & QRS-peak & QRS-peak & QRS-peak/Full \\
& & & & & &
\end{tabular}

the MSP430F5437A and assuming a $3.7 \mathrm{~V}$ and $450 \mathrm{mAh}$ battery. Actually, power consumption will be higher due to other modules (e. g. radio module), but what it is analyzed here is just the CPU consumption due to the execution of the algorithm.

For Standard and High-accuracy modes two different values are presented for the computational burden and RAM and Flash memories. The lowest values belong to single QRS peaks detection, for which the On-end module of the algorithm is disabled, and the highest values correspond to a full delineation. These are the upper and lower limits of the range in which the modes can operate with different combinations of the algorithm modules.

Nowadays, the power consumption of portable electronic devices is an important matter to be taken into account, since they operate with a limited battery supply. Thus, extending the battery life of such devices is crucial in many situations. In High-accuracy mode, the average computational burden for a full delineation is $8.5 \%$, which means that the algorithm needs an average of 2733 cycles per new sample, since the sampling rate is $250 \mathrm{~Hz}$ and the microcontroller has 32000 available cycles between samples acquisition. The memory footprint used is $9 \mathrm{kB}$ of Flash memory and $9 \mathrm{kB}$ of RAM.

The computational burden is divided in three main tasks: $50 \%$ for the FIR filtering, $40 \%$ for the morphological filtering and $10 \%$ for the rest of the delineation. Therefore, the signal filtering (FIR and morphological filters) is the most time-demanding process and has been carefully developed for optimizing the results.

The FIR filters stage has been optimized using symmetry criteria for the filter coefficients and reducing flash memory accesses implementing both FIR filters at the same time. Two main optimizations have been done for reducing execution time for the MSP430. First, it is mandatory to avoid floating-point arithmetic in this type of low-power MCUs. A good solution for FIR filtering is the coefficient quantization, that consists of converting the floating point coefficients to integer coefficients, multiplying each coefficient $b_{i}$ by a power of 2 and rounding $\left(B_{i}=\operatorname{round}\left(b_{i} \cdot 2^{b}\right)\right)$. It must be taken into account that this operation introduces an error (the difference between the value in the output and the real value) given by $e_{i, \max }=2^{-b-1}$ [43] and that output signal is amplified. In our case, the coefficients have been multiplied by $1024(b=10)$ therefore the error is given by $2^{-11}=4.9 \cdot 10^{-4}$, which is an assumable error in order to avoid floating-point arithmetic.

When a FIR filter operation is being done, the output bit growth, $B \leq \log _{2} \sum\left|B_{i}\right|$ [44], must be taken into account in order to avoid overflows. The multiply-accumulate operation requires a bigger word size than the input samples word size to avoid overflow. For an order-40 filter, the bit growth of both filters $\left(f_{c}=14 \mathrm{~Hz}\right.$ and $\left.f_{c}=40 \mathrm{~Hz}\right)$ is $B \leq 11$ bits, therefore if the input samples are 12-bit integer, the maximum word size necessary for the output is 23 bits, forcing to use a 32 bit accumulator. For smaller orders, the bit growth is smaller. Due to the 16-bit architecture of the MSP430F5437A, multiplications and additions are not native for 32-bit operands, in consequence, the number of instructions needed to perform them is incremented. The computational burden devoted to the FIR filtering would be strongly reduced using a 32-bit microcontroller.

As it can be seen in Table $\mathrm{V}$ and thanks to the modularity of the algorithm, the Shimmer3 would consume $1145 \mu \mathrm{W}$ to perform a complete delineation at $250 \mathrm{~Hz}$ sampling rate using the High-accuracy mode ( $475 \mu \mathrm{W}$ due to the CPU and 670 $\mu \mathrm{W}$ due to the ADS) and $765 \mu \mathrm{W}$ to perform only QRS peaks detection using the Standard mode ( $95 \mu \mathrm{W}$ due to the CPU and $670 \mu \mathrm{W}$ due to the ADS), achieving 33\% power saving with a marginal delineation quality degradation.

The previous wavelet approach work [21] showed similar computational burden and memory footprint than the Highaccuracy mode using the same platform, but without the possibility of being adaptive to different scenarios, reducing the power consumption if wanted.

The other feature already presented of this algorithm with respect to other techniques, is that this algorithm can be easily adapted to different ECG sampling rates. Obviously, the higher the sampling rate, the more the computational burden because more ECG samples are received per second. If the sampling rate is increased by a $n$ factor $\left(f_{s}^{*} \approx n \cdot f_{s}\right)$, the computational burden is multiplied by $n$ for the FIR filtering, neither the number of operations per sample nor the memory footprint changes. For the morphological filters, the number of operations per new sample remains more or less the same and the memory footprint is also multiplied by $n$, because the size of the used buffers depends directly on the sampling rate (the size is time-domain defined).

The Ultra-low-power and Low-power modes work at $50 \mathrm{~Hz}$ ECG sampling rate. This involves that the computational burden is reduced to only $0.4 \%$ in the Low-power mode and $0.2 \%$ in the Ultra-low-power mode, reducing power consumption of the Shimmer 3 to $445 \mu \mathrm{W}$ (30 $\mu \mathrm{W}$ due to the CPU and $415 \mu \mathrm{W}$ due to the ADS) and $315 \mu \mathrm{W}(15 \mu \mathrm{W}$ due to the CPU and $300 \mu \mathrm{W}$ 
due to the ADS) respectively. In the latter case, the ADS remains in sleep-mode during $73 \%$ of the time achieving $70 \%$ of power saving with respect to a complete delineation in High-accuracy mode.

In this way, the battery life of devices is remarkably increased by reducing the computational burden. For instance. in healthy patients, Ultra-low-power mode can be used regularly, while no arrhythmia is present. However, if any arrhythmia is detected, a complete delineation can be performed switching to Highaccuracy mode in order to collect more information during a given period of time, this mode is denoted as Adaptive mode, see Fig. 11. The results delineating the QT database in Adaptive mode show that the system runs in Ultra-low-power mode $87 \%$ of the time (61\% Discontinuous sampling and 26\% Continuous sampling) and the Standard or High-accuracy modes $13 \%$ of the time, with an associated mean power consumption of $423 \mu \mathrm{W}$. Therefore, if just data acquisition and sample processing are considered for the Shimmer3, the lifetime of the system would be 23.5 weeks, in which the patient will be monitored using an Ultra-low-power mode when heart rate is stable and with a High-accuracy mode otherwise.

\section{CONCLUSION}

In this paper, a new simple, modular and effective algorithm has been developed to delineate and locate the peaks and boundaries of the different ECG waves, such as the P wave, the QRS complex and the $\mathrm{T}$ wave, featuring low computational cost and mathematical complexity while achieving similar or better accuracy than other more advanced and computationally intensive state-of-the-art techniques. The algorithm is conceived as a modular design, in which the Peaks module delineates the ECG peaks, computing the first and second derivatives of an aggressive low-pass filtered signal, and the On-end module detects the onsets and ends of the peaks, using a less aggressive low-pass filter and morphological filters.

To validate the performance of the proposed algorithm, the QT and MIT-BIH databases have been delineated and the results have been compared with annotations made by cardiologists for these databases. Results show sensitivity higher than $98 \%$ when detecting the ECG wave peaks and $96 \%$ for onsets and ends, and better positive predictive value in contrast to more complex techniques as, for example, wavelets or fasor transform. Moreover, the errors in the delineation of all the fiducial points are below the tolerances given by the Common Standards for Electrocardiography (CSE) committee, except for the $\mathrm{P}$ wave onset, for which the algorithm is above the agreed tolerances by only a fraction of the sample duration.

The presented algorithm has the advantage of being highly adaptive and modular. The system is able to automatically modify the sampling rate or to disable some modules of the algorithm such as for $\mathrm{P}$ or $\mathrm{T}$ wave detectors, or the detection of onsets and ends of the ECG waves, depending whether a complete delineation is needed, because an abnormal cardiac event is detected, or performing a reduced delineation the rest of the time for energy saving. This flexibility allows the algorithm to be able to operate in different modes dealing with the trade- off between delineation accuracy and energy consumption. The proposed technique has been implemented in a simulated commercial WBSN platform to analyze its computational burden and memory footprint showing that it can perform a complete high-accuracy real-time ECG delineation with a computational burden of $8.5 \%$ (8.6 weeks of battery lifetime just considering data acquisition and processing), similar to the wavelet approach in [21], and also a real-time detection of only the QRS peaks, reducing the computational load to only $0.2 \%$ (31 weeks of battery lifetime) and achieving $70 \%$ power saving with respect to a complete delineation.

The system can also be configured to automatically switch between modes, denoted as Adaptive mode, in order to perform a full high-accuracy delineation during arrhythmias and a limited ultra-low power detection when no arrhythmia is detected. This mode has a combined computational load of $1.3 \%$ (21.7 weeks of battery lifetime).

Its low complexity, modularity in the delineation, low-power consumption, as well as its easy and quick adaptability to the ECG sampling rate, offer a great flexibility and qualify the proposed algorithm as an useful tool for embedded medical devices with limited resources for real-time applications.

\section{REFERENCES}

[1] J. Pan and W. J. Tompkins, "A real-time QRS detection algorithm," IEEE Trans. Biomed. Eng., vol. BME-32, no. 3, pp. 230-236, Mar. 1985.

[2] V. Bono et al., "Development of an automated updated selvester QRS scoring system using SWT-based QRS fractionation detection and classification," IEEE J. Biomed. Health Informat., vol. 18, no. 1, pp. 193-204, Jan. 2014.

[3] C. Li, C. Zheng, and C. Tai, "Detection of ECG characteristic points using wavelet transforms," IEEE Trans. Biomed. Eng., vol. 42, no. 1, pp. 21-28, Jan. 1995.

[4] J. Sahambi, S. Tandon, and R. Bhatt, "Using wavelet transforms for ECG characterization. An on-line digital signal processing system," IEEE Eng. Med. Biol. Mag., vol. 16, no. 1, pp. 77-83, Jan./Feb. 1997.

[5] J. P. Martínez, R. Almeida, S. Olmos, A. P. Rocha, and P. Laguna, "A wavelet-based ECG delineator: Evaluation on standard databases," IEEE Trans. Biomed. Eng., vol. 51, no. 4, pp. 570-581, Apr. 2004.

[6] D. Benitez, P. Gaydecki, A. Zaidi, and A. Fitzpatrick, "The use of the hilbert transform in ECG signal analysis," Comput. Biol. Med., vol. 31, no. 5, pp. 399-406, 2001.

[7] A. Martinez, R. Alcaraz, and J. J. Rieta, "Automatic electrocardiogram delineator based on the phasor transform of single lead recordings," in Proc. Comput. Cardiol., 2010, pp. 987-990.

[8] H. Vullings, M. Verhaegen, and H. Verbruggen, "Automated ECG segmentation with dynamic time warping," in Proc. 20th Annu. Int. Conf IEEE Eng. Med. Biol. Soc., Oct. 1998, vol. 1, pp. 163-166.

[9] Z. Dokur, T. Olmez, M. Korurek, and E. Yazgan, "Detection of ECG waveforms by using artificial neural networks," in Proc. 18th Annu. Int. Conf. IEEE Eng. Med. Biol. Soc., Oct. 1996, vol. 3, pp. 929-930.

[10] S. Graja and J.-M. Boucher, "Hidden markov tree model applied to ecg delineation," IEEE Trans. Instrum. Meas., vol. 54, no. 6, pp. 2163-2168, Dec. 2005

[11] E. B. Mazomenos, T. Chen, A. Acharyya, A. Bhattacharya, J. Rosengarten, and K. Maharatna, "A time-domain morphology and gradient based algorithm for ECG feature extraction," in Proc. IEEE Int. Conf. Ind. Technol., Mar. 2012, pp. 117-122.

[12] Y. Sun, K. Luk Chan, and S. Muthu Krishnan, "Characteristic wave detection in ECG signal using morphological transform," BMC Cardiovascular Disorders, vol. 5, no. 1, 2005, Art. no. 28.

[13] Corventis NUVANT Mobile Cardiac Telemetry System, 2017. [Online]. Available: http://www.corventis.com/

[14] Vital Connect HealthPatch MD, 2017. [Online]. Available: http://www. vitalconnect.com/

[15] Preventice BodyGuardian Remote Monitoring System, 2017. [Online]. Available: http://www.preventice.com/ 
[16] Fitbit, 2017. [Online]. Available: https://www.fitbit.com/

[17] Garmin, Vívo-Series, 2017. [Online]. Available: https://explore.garmin. com/en-US/vivo-fitness/

[18] Polar, M400, 2017. [Online]. Available: https://www.polar.com/en/ products/sport/M400

[19] Cronovo, 2017. [Online]. Available: http://www.cronovo.com/

[20] QardioCore, 2017. [Online]. Available: https://www.getqardio.com/ qardiocore-wearable-ecg-ekg-monitor-iphone/

[21] F. Rincón, J. Recas, N. Khaled, and D. Atienza, "Development and evaluation of multilead wavelet-based ECG delineation algorithms for embedded wireless sensor nodes," IEEE Trans. Inf. Technol. Biomed., vol. 15, no. 6, pp. 854-863, Nov. 2011.

[22] R. Braojos Lopez, G. Ansaloni, and D. Atienza Alonso, "A methodology for embedded classification of heartbeats using random projections," in Proc. Des. Autom. Test Europe Conf., 2013, pp. 899-904.

[23] N. Bayasi, T. Tekeste, H. Saleh, B. Mohammad, A. Khandoker, and M. Ismail, "Low-power ECG-based processor for predicting ventricular arrhythmia," IEEE Trans. Very Large Scale Integr. Syst., vol. 24, no. 5, pp. 1962-1974, May 2016.

[24] S. A. Guidera and J. S. Steinberg, "The signal-averaged P wave duration: A rapid and noninvasive marker of risk of atrial fibrillation," J. Amer. Coll. Cardiol., vol. 21, no. 7, pp. 1645-1651, 1993.

[25] C. Saritha, V. Sukanya, and Y. N. Murthy, "ECG signal analysis using wavelet transforms," Bulgarian J. Phys., vol. 35, no. 1, pp. 68-77, 2008.

[26] R. B. Northrop, Noninvasive Instrumentation and Measurement in Medical Diagnosis. Boca Raton, FL, USA: CRC Press, 2001.

[27] J. Van Alste and T. Schilder, "Removal of base-line wander and powerline interference from the ECG by an efficient FIR filter with a reduced number of taps," IEEE Trans. Biomed. Eng., vol. BME-32, no. 12, pp. 1052-1060, Dec. 1985.

[28] Y. Sun, K. L. Chan, and S. M. Krishnan, "ECG signal conditioning by morphological filtering," Comput. Biol. Med., vol. 32, no. 6, pp. 465-479, 2002.

[29] M. Gertsch, The ECG: A Two-Step Approach to Diagnosis. New York, NY, USA: Springer, 2003.

[30] P. Laguna, R. G. Mark, A. Goldberg, and G. B. Moody, "A database for evaluation of algorithms for measurement of QT and other waveform intervals in the ECG," in Proc. Comput. Cardiol., 1997, pp. 673-676.
[31] A. L. Goldberger et al., "Physiobank, physiotoolkit, and physionet components of a new research resource for complex physiologic signals," Circulation, vol. 101, no. 23, pp. e215-e220, 2000.

[32] I. Silva and G. Moody, "An open-source toolbox for analysing and processing PhysioNet databases in MATLAB and Octave," J. Open Res. Soft., vol. 2, no. 1, p. e27, 2014.

[33] Testing and Reporting Performance Results of Cardiac Rhythm and ST Segment Measurement Algorithms. NSI/AAMI EC57:1998/(R)2008 (Revision of AAMI ECAR:1987), Association for the Advancement of Medical Instrumentation, 1999.

[34] L. Y. Di Marco and L. Chiari, "A wavelet-based ECG delineation algorithm for 32-bit integer online processing," Biomed. Eng. Online, vol. 10, no. 1, 2011, Art. no. 23.

[35] The CSE Working Party, "Recommendations for measurement standards in quantitative electrocardiography," Eur. Heart J., vol. 6, no. 10, pp. 815-825, 1985.

[36] S. Mehta and N. Lingayat, "Detection of P and T-waves in electrocardiogram," in Proc. World Congr. Eng. Comput. Sci., 2008, pp. 22-24.

[37] Texas Instruments, ADS1292, 2017. [Online]. Available: http://www. ti.com/product/ADS1292

[38] G. B. Moody and R. G. Mark, "The impact of the MIT-BIH arrhythmia database," IEEE Eng. Med. Biol. Mag., vol. 20, no. 3, pp. 45-50, May/Jun. 2001.

[39] G. B. Moody and R. G. Mark, "The MIT-BIH Arrhythmia Database on CD-ROM and software for use with it," in Proc. Comput. Cardiol., 1990, pp. $185-188$.

[40] E. B. Mazomenos et al., "A low-complexity ECG feature extraction algorithm for mobile healthcare applications," IEEE J. Biomed. Health Informat., vol. 17, no. 2, pp. 459-469, Mar. 2013.

[41] Shimmer, 2017. [Online]. Available: http://www.shimmersensing.com

[42] J. Eriksson, A. Dunkels, N. Finne, F. Osterlind, and T. Voigt, "Mspsim - an extensible simulator for msp430-equipped sensor boards," in Proc. Eur. Conf. Wireless Sens. Netw., 2007, p. 27.

[43] R. Yates, Practical Considerations in Fixed-Point FIR Filter Implementations, Digital Signal Labs, Technical Reference, Mar. 2010.

[44] IP LogiCORE FIR Compiler v5.0, Xilinx, San Jose, CA, USA, 2011. 